\title{
Research Article \\ Guaranteed Performance Robust Kalman Filter for Continuous-Time Markovian Jump Nonlinear System with Uncertain Noise
}

\author{
Jin Zhu, Junhong Park, Kwan-Soo Lee, and Maksym Spiryagin \\ Department of Mechanical Engineering, Hanyang University, 17 Haengdang-dong, Seongdong-gu, \\ Seoul 133-791, South Korea \\ Correspondence should be addressed to Jin Zhu, jinzhu@ustc.edu
}

Received 29 January 2008; Accepted 13 July 2008

Recommended by Paulo Gonçalves

Robust Kalman filtering design for continuous-time Markovian jump nonlinear systems with uncertain noise was investigated. Because of complexity of Markovian jump systems, the statistical characteristics of system noise and observation noise are time-varying or unmeasurable instead of being stationary. In view of robust estimation, maximum admissible upper bound of the uncertainty to noise covariance matrix was given based on system state estimation performance. As long as the noise uncertainty is limited within this bound via noise control, the Kalman filter has robustness against noise uncertainty, and stability of dynamic systems can be ensured. It is proved by game theory that this design is a robust mini-max filter. A numerical example shows the validity of this design.

Copyright (C) 2008 Jin Zhu et al. This is an open access article distributed under the Creative Commons Attribution License, which permits unrestricted use, distribution, and reproduction in any medium, provided the original work is properly cited.

\section{Introduction}

Optimal filtering problem has been a hot topic in past decades, within which Kalman filtering is one of the most popular estimation approaches and considerable effort has been devoted to its theory and applications. The applications of Kalman filtering theory may be found in a large spectrum of different fields ranging from various engineering problems to biology, geoscience, economics, and management [1]. For standard Kalman filtering, one of the key assumptions is that system noise and observation noise are Gaussian, whose covariances are known and stationary. However, in many actual problems, the statistical characteristics (covariances) of noise may be time-varying instead of being stationary, and in some cases it is impossible to get the exact measurement values, which means that the noise covariances are uncertain instead of being exactly known; for this reason, the stochastic noise is called "uncertain." Consequently, the standard Kalman filter may not be robust 
against modeling uncertainty and disturbances. Thus, the study of a robust state estimation approach is of practical importance and has been attracting more interest over the past few years. A useful approach is to use a game-theoretic formulation with which one minimizes the worst performance stimulated by uncertain factors, and some corresponding results of robust filtering for linear systems with uncertain noise have been addressed in [2-4].

On the other hand, Markovian jump systems, which are convenient tools for representing many real-world systems [5], have aroused much attention in recent years. In the case of fault detection, fault-tolerant control, and multimodal control, discrete jumps in the continuous dynamics are used to model component failures and sudden switch of system dynamics. With further study of Markovian jump systems, many achievements have been made in the last decade on stability analysis [6,7], filtering [8,9], and controller design [10, 11]. Among the efforts towards filtering, Shi et al. [12] and Mahmoud et al. [13] gave Kalman filtering equations for continuous-time and discrete-time Markovian jump linear systems with structure uncertainty, respectively. However, in the above-referred contributions, all the research work is facing the same problem as that of nonjump systems; both the state equation and output measurement are subjected to stationary Gaussian noise so that an optimal filtering gain is obtained based on the stationary noise covariance matrix. But this is only an ideal assumption for Markovian jump systems. As we know, Markovian jump systems are used to represent a class of systems which are usually accompanied by sudden changes of working environment or system dynamics. For this reason, noise uncertainty occurs more frequently or with more probability than in nonjump systems. Moreover, with the uncertainty to noise covariance matrix increasing, the estimation of system state tends to be inaccurate or false, which may cause errors in control signals and in worst case may lead to breakdown of the whole dynamic systems.

To avoid this tragical situation, a direct way dealing with this problem is to redesign Kalman filter for jump systems by using new noise covariance matrix. But as we have pointed out above, it is almost impossible to get the real-time information of noise covariance matrix since it is time-varying or unmeasurable; therefore we could not update Kalman filter gain online. Another feasible way is to give an admissible bound for estimation performance of system state so that the predesigned Kalman filter will remain effective and the system operates in the course of nature as long as the real-time estimation error is within this precision. To achieve this purpose, we perform the following design method. By using the view of robust estimation, a maximum bound of noise covariance matrix uncertainty is obtained through calculation according to admissible bias for estimation performance of system state. If we could ensure the noise uncertainty to be within this bound via technical method such as noise control, the estimation of system state can be within a desired precision, and thus stability of the whole dynamic process can be achieved. It should be noted that in this research work, we do not mean to eliminate the effect of noise entirely because it is almost impossible or highly costly to do so in practical environment. Our work focuses on the upper bound of noise change level; thus it means only that the change to noise covariance matrix is required to be limited within this bound no matter what the stationary covariance matrix is.

In this paper, robust Kalman filtering for continuous-time Markovian jump nonlinear systems with uncertain noise is considered. Firstly, we give some assumptions so that the nonlinear jump systems can be modeled as a linear one by local linearization. Secondly, we seek the maximum upper bound of nonstructural uncertainty to noise covariance matrix such that the deviation of performance can be within a prescribed precision. Then, we discuss the analytical solution of maximum bound using Lagrange method. Finally, we prove the establishment of saddle inequality, and show that this filter design is a mini-max robust 
filter using game theory. At the end of the paper, an illustrative example is used to show the validity of our method.

\section{Problem Description}

Throughout the paper, unless otherwise specified, we denote by $\left(\Omega, \mathcal{F},\left\{\mathcal{F}_{t}\right\}_{t \geq 0}, \mathbf{P}\right)$ a complete probability space with filtration $\left\{\mathcal{F}_{t}\right\}_{t \geq 0}$ satisfying the usual conditions (i.e., it is rightcontinuous, and $\mathcal{F}_{0}$ contains all $p$-null sets). Let $|x|$ stand for the usual Euclidean norm for a vector $x$, and let $|X|$ denote the Frobenius norm of a matrix $X$ defined by $|X|=\lambda_{\max }^{1 / 2}\left(X X^{T}\right)$, where $\lambda_{\max }(\cdot)$ is the maximum eigenvalue of matrix and the superscript $T$ represents transpose. Operator $\operatorname{Tr}(\cdot)$ denotes the matrix trace, and we denote by $X>0(\geq 0)$ that matrix $X$ is positive definite (semipositive definite). Let $\{r(t), t \geq 0\}$ be a right-continuous Markov process on the probability space taking values in finite state space $S=\{1,2, \ldots, N\}$ with $\Pi=\left[\pi_{i j}\right]$ being the chain generator, an $N \times N$ matrix. The entries $\pi_{i j}, i, j \in S$, are interpreted as transition rates such that

$$
\mathbf{P}(r(t+d t)=j \mid r(t)=i)= \begin{cases}\pi_{i j} d t+o(d t) & \text { if } i \neq j, \\ 1+\pi_{i j} d t+o(d t) & \text { if } i=j,\end{cases}
$$

where $d t>0$ and $\lim _{d t \rightarrow 0}(o(d t) / d t)=0$. Here, $\pi_{i j}>0(i \neq j)$ is the transition rate from $i$ to $j$. Notice that the total probability axiom imposes $\pi_{i i}<0$ and

$$
\sum_{j=1}^{N} \pi_{i j}=0, \quad \forall i \in S
$$

Consider the following continuous-time Markovian jump nonlinear system with uncertain noise:

$$
\begin{aligned}
& \dot{x}=f(x, r(t))+\omega^{0}, \\
& y=h(x, r(t))+v^{0},
\end{aligned}
$$

where $x \in \mathbf{R}^{n}$ is state vector, and $y \in \mathbf{R}^{m}$ is measurement output. $f(\cdot, \cdot) \in \mathbf{R}^{n}$ and $h(\cdot, \cdot) \in \mathbf{R}^{m}$ are nonlinear vector functions. $\omega^{0}$ and $v^{0}$ are $n$-dimensional and $m$-dimensional white noises and satisfy the following assumption.

Assumption 2.1. For any given time $s, t \geq 0$, there are

(1) $E\left[\omega_{t}^{0}\right]=0, E\left[v_{t}^{0}\right]=0$,

(2) $\operatorname{Cov}\left[\omega_{t}^{0}, \omega_{s}^{0}\right]=W^{0} \delta_{t, s}=(W+\Delta W) \delta_{t, s}, W \geq 0, \Delta W \geq 0$,

(3) $\operatorname{Cov}\left[v_{t}^{0}, v_{s}^{0}\right]=V^{0} \delta_{t, s}=(V+\Delta V) \delta_{t, s}, V>0, \Delta V \geq 0$,

(4) $E\left[\left(\begin{array}{c}w_{t}^{0} \\ v_{t}^{0}\end{array}\right) \cdot\left(w_{s}^{0 T} v_{s}^{0 T}\right)\right]=\left[\begin{array}{cc}W^{0} \delta_{t, s} & 0 \\ 0 & V^{0} \delta_{t, s}\end{array}\right]$.

In Assumption 2.1, $W^{0} \in \mathbf{R}^{n \times n}$ and $V^{0} \in \mathbf{R}^{m \times m}$ consist of two parts, where $W$ and $V$ denote the stationary noise covariance matrix, whose values are exactly known. $\Delta W$ and $\Delta V$ denote the noise uncertainty caused by time-varying or sudden switch of system dynamics; they are unknown but norm-bounded. $\delta(\cdot, \cdot)$ is a Dirac function taking values in $\{0,1\}$. For the deduction of robust Kalman filter, we introduce the following assumption. 
Assumption 2.2. For any fixed system mode $r(t)=i \in S$ and vector $\sigma \in \mathbf{R}^{n}$, the nonlinear vector functions $f(\cdot, \cdot), h(\cdot, \cdot)$ are assumed to satisfy $f(0, i)=h(0, i)=0$ and

$$
\begin{gathered}
|f(x+\sigma, i)-f(x, i)-A(i) \sigma| \leq|\Delta A(i)||\sigma|, \\
|h(x+\sigma, i)-h(x, i)-C(i) \sigma| \leq|\Delta C(i)||\sigma|,
\end{gathered}
$$

where $A(i), C(i)$ are Jacobian matrices of $f(\cdot, \cdot), h(\cdot, \cdot)$, and $\Delta A(i), \Delta C(i)$ satisfy

$$
\begin{aligned}
& \Delta A(i)=H_{1}(i) F(i) E(i), \\
& \Delta C(i)=H_{2}(i) F(i) E(i),
\end{aligned}
$$

where $H_{1}(i), H_{2}(i)$, and $E(i), i \in S$, are known constant matrices, and $F(i), i \in S$, is unknown matrix satisfying $F^{T}(i) F(i) \leq I$. Establishing Assumption 2.2, the Markovian jump nonlinear system could be transformed to a nominal linear model via local linearization technique:

$$
\begin{aligned}
& \dot{x}=[A(r(t))+\Delta A(r(t))] x+\omega^{0}, \\
& y=[C(r(t))+\Delta C(r(t))] x+v^{0} .
\end{aligned}
$$

For simplification, we denote $A(r(t)=i), H_{1}(r(t)=i), H_{2}(r(t)=i), E(r(t)=i), \Delta A(r(t)=$ $i), C(r(t)=i)$, and $\Delta C(r(t)=i)$ by $A_{i}, H_{1 i}, H_{2 i}, E_{i}, \Delta A_{i}, C_{i}$, and $\Delta C_{i}$.

Theorem 2.3. Consider stochastically stable Markovian jump system (2.6), and assume that the noise is stationary, which means that $\Delta W=\Delta V=0$. Then, one has the following standard Kalman filter (see [12]):

$$
\dot{\hat{x}}=\widehat{A}_{i} \widehat{x}+K_{i}\left[y-\widehat{C}_{i} \widehat{x}\right],
$$

where filtering gain $K_{i}$ is given by the following coupled Riccati equations:

$$
\begin{gathered}
\widehat{A}_{i}=A_{i}+\left(\frac{1}{\epsilon_{i}} H_{1 i} H_{1 i}^{T}+W\right) P_{i}^{-1}, \\
\widehat{C}_{i}=\frac{1}{\epsilon_{i}} H_{2 i} H_{1 i} P_{i}^{-1}+C_{i} \\
A_{i} P_{i}+P_{i} A_{i}^{T}+\epsilon_{i} P_{i} E_{i}^{T} E_{i} P_{i}+\sum_{j=1}^{N} \pi_{i j} P_{j}+\frac{1}{\epsilon_{i}} H_{1 i} H_{1 i}^{T}+W=0, \\
K_{i}=\left(Q_{i} \widehat{C}_{i}^{T}+\frac{1}{\epsilon_{i}} H_{1 i} H_{2 i}^{T}\right)\left(\frac{1}{\epsilon_{i}} H_{2 i} H_{2 i}^{T}+V\right)^{-1}, \\
\left(\widehat{A}_{i}-K_{i} \widehat{C}_{i}\right) Q_{i}+Q_{i}\left(\widehat{A}_{i}-K_{i} \widehat{C}_{i}\right)^{T}+K_{i} V K_{i}^{T}+\sum_{j=1}^{N} \pi_{i j} Q_{j}+\frac{1}{\epsilon_{i}} H_{1 i} H_{1 i}^{T}+W=0 .
\end{gathered}
$$

Here, matrices $P_{i}>0, Q_{i}>0$ and scalar $\epsilon_{i}$ are chosen so that $\operatorname{tr}\left(Q_{i}\right)$ reaches the minimum.

With the above standard Kalman filter gain $K_{i}$ adopted, the state estimation error is

$$
E\left\{(x-\widehat{x})^{T}(x-\widehat{x})\right\} \leq \max _{j \in S} \operatorname{tr}\left(Q_{j}\right) .
$$




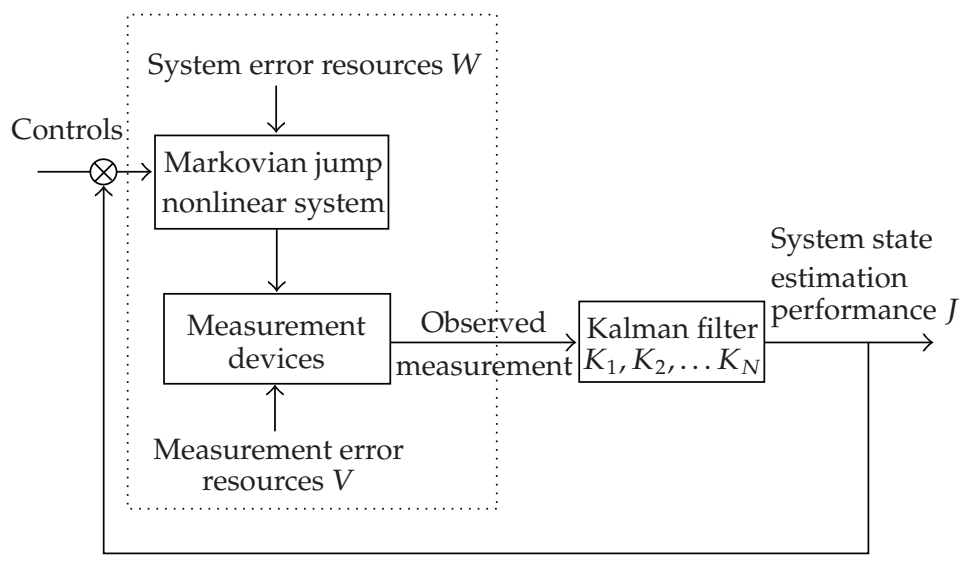

Figure 1: Standard Kalman filter with stationary noise.

Define the estimation error performance as

$$
J\left(K_{1}, K_{2}, \ldots, K_{N}, W, V\right)=\max _{j \in S} \operatorname{tr}\left(Q_{j}\right) .
$$

According to Theorem 2.3 and the quality of Kalman filtering, if the noise is stationary $(\Delta W=$ $\Delta V=0)$, the estimation error performance can achieve the minimum value by adopting standard Kalman filtering (2.7).

Now, we consider that the noise is not stationary, which means that $\Delta W \neq 0$ and $\Delta V \neq 0$; thus the new covariance matrix of noise is $W^{0}, V^{0}$. If we still adopt the former predesigned standard Kalman filter gain $K_{i}$, the new state estimation error should be $Q_{i}^{0}$, which satisfies

$$
\left(\widehat{A}_{i}-K_{i} \widehat{C}_{i}\right) Q_{i}^{0}+Q_{i}^{0}\left(\widehat{A}_{i}-K_{i} \widehat{C}_{i}\right)^{T}+K_{i} V^{0} K_{i}^{T}+\sum_{j=1}^{N} \pi_{i j} Q_{j}^{0}+\frac{1}{\epsilon_{i}} H_{1 i} H_{1 i}^{T}+W^{0}=0
$$

Therefore, the new estimation error performance is

$$
J\left(K_{1}, K_{2}, \ldots, K_{N}, W^{0}, V^{0}\right)=\max _{j \in S} \operatorname{tr}\left(Q_{j}^{0}\right) .
$$

According to (2.10) and (2.12), the deviation of estimation error performance yielded by noise uncertainty $(\Delta W, \Delta V)$ can be written as

$$
\begin{aligned}
\Delta J\left(K_{1}, K_{2}, \ldots, K_{N}, \Delta W, \Delta V\right) & =J\left(K_{1}, \ldots, K_{N}, W^{0}, V^{0}\right)-J\left(K_{1}, \ldots, K_{N}, W, V\right) \\
& =\max _{j \in S} \operatorname{tr}\left(Q_{j}^{0}\right)-\max _{j \in S} \operatorname{tr}\left(Q_{j}\right) \leq r,
\end{aligned}
$$

where $r>0$ is a parameter which is given according to detailed precision request of practical dynamic process.

Our design purpose is shown in Figures 1 and 2. Suppose that the noise is stationary with covariance matrix $W, V$, and that the system filtering performance is $J$ with standard filtering gain $K_{i}$ adopted as shown in Figure 1. But now noise is with uncertainty $(\Delta W, \Delta V)$; 


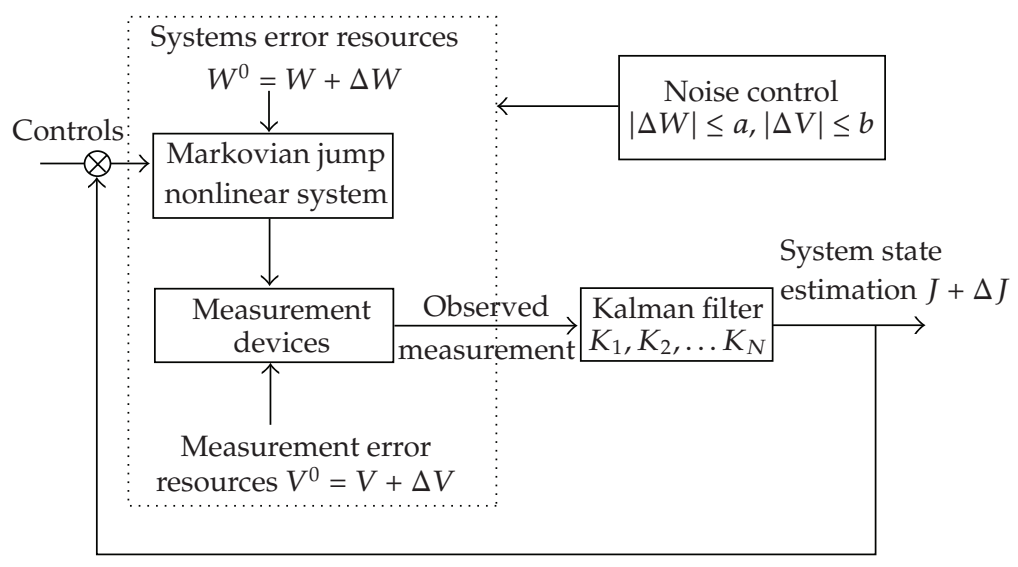

Figure 2: Robust Kalman filter with uncertain noise.

the former designed Kalman filter gain $K_{i}$ will no longer be an optimal one. If we still want to get the precise estimation of system state so that the dynamic system could remain stable, there are two choices. One is to update the Kalman filter gain $K_{i}$ according to new noise covariance matrix $(W+\Delta W, V+\Delta V)$, but this way is impossible or highly costly. Another way is to still adopt the former designed filtering gain $K_{i}$ and take some actions in noise control. Based on this idea, the new estimation performance is $J+\Delta J$ with $K_{i}$ adopted, and a deviation $\Delta J$ occurs resulting from noise uncertainty (see Figure 2). For the robustness of system, which means that $\Delta J$ is less than an admissible precision $r$, there must besome limitation to noise uncertainty. Using the view of robust estimation, we are trying to find a maximum upper bound $a, b$ for the uncertainty to noise covariance matrix. As long as the noise uncertainty is controlled to satisfy $|\Delta W| \leq a,|\Delta V| \leq b$ via noise control, we will achieve deviation of estimation performance to be within the admissible precision $r$, which means that $\Delta J \leq r$; thus the general system has robustness to noise uncertainty, and stability of the whole dynamic process can be maintained whatever the original stationary noise covariance matrix $(W, V)$ is. In the following part of this paper, we seek the solution of maximum upper bound $a, b$.

\section{Upper Bound of Nonstructural Noise Uncertainty}

\subsection{Mathematical Expression of Upper Bound}

According to (2.8) and (2.11), we have

$$
\left(\widehat{A}_{i}-K_{i} \widehat{C}_{i}\right) \Delta Q_{i}+\Delta Q_{i}\left(\widehat{A}_{i}-K_{i} \widehat{C}_{i}\right)^{T}+\sum_{j=1}^{N} \pi_{i j} \Delta Q_{j}+\Delta W+K_{i} \Delta V K_{i}^{T}=0
$$

where $\Delta Q_{i}=Q_{i}^{0}-Q_{i}$. From the above equation, it is easily seen that $\operatorname{tr}\left(\Delta Q_{i}\right)$ is a linear mapping of $(\Delta W, \Delta V)$. Define a compact convex set as $\Xi=\left\{(\Delta W, \Delta V): 0 \leq \Delta W \leq \Delta W^{*}, 0 \leq\right.$ $\left.\Delta V \leq \Delta V^{*}\right\}$; thus the deviation of performance $\Delta J\left(K_{1}, K_{2}, \ldots, K_{N}, \Delta W, \Delta V\right)$ is a mapping from $\Xi$ to $\mathbf{R}^{1}$, and it has the following facts. 
Fact 1. For any given noise uncertainty $\left(\Delta W_{j}, \Delta V_{j}\right) \in \Xi, j=1,2$, if $\Delta W_{1} \leq \Delta W_{2}$ and $\Delta V_{1} \leq$ $\Delta V_{2}$, one has

$$
\Delta J\left(K_{1}, K_{2}, \ldots, K_{N}, \Delta W_{1}, \Delta V_{1}\right) \leq \Delta J\left(K_{1}, K_{2}, \ldots, K_{N}, \Delta W_{2}, \Delta V_{2}\right) .
$$

Fact 2. Define the maximum admissible deviation of estimation performance $r$ as

$$
r=\max _{(\Delta W, \Delta V) \in \Xi} \Delta J\left(K_{1}, K_{2}, \ldots, K_{N}, \Delta W, \Delta V\right) .
$$

Thus, $r$ could be achieved only by maximum noise uncertainty $\left(\Delta W^{*}, \Delta V^{*}\right)$, which means that

$$
r=\Delta J\left(K_{1}, K_{2}, \ldots, K_{N}, \Delta W^{*}, \Delta V^{*}\right) .
$$

The purpose of the following work is to construct a maximal compact convex set $\Xi^{*}$, as long as the noise uncertainty satisfies $(\Delta W, \Delta V) \in \Xi^{*},(2.13)$ is sure to establish. According to the finity of mode $S,(2.13)$ is equivalent to

$$
\operatorname{tr}\left(Q_{i}^{0}\right) \leq r+\max _{j \in S} \operatorname{tr}\left(Q_{j}\right)
$$

Therefore, for each mode $i \in S$, there is

$$
\operatorname{tr}\left(\Delta Q_{i}\right)=\operatorname{tr}\left(Q_{i}^{0}\right)-\operatorname{tr}\left(Q_{i}\right) \leq r+\max _{j \in S} \operatorname{tr}\left(Q_{j}\right)-\operatorname{tr}\left(Q_{i}\right) .
$$

Define the maximum upper bound of noise uncertainty as $|\Delta W| \leq a,|\Delta V| \leq b$; thus

$$
0 \leq \Delta W \leq a I_{n}, \quad 0 \leq \Delta V \leq b I_{m}
$$

Substituting the above inequalities into (3.1) and (3.6), one has

$$
a \operatorname{tr}\left(D_{i}\right)+b \operatorname{tr}\left(G_{i}\right) \leq r+\max _{j \in S} \operatorname{tr}\left(Q_{j}\right)-\operatorname{tr}\left(Q_{i}\right), \quad \forall i \in S,
$$

where matrices $D_{i}, G_{i}>0, i \in S$, satisfy the following coupled Riccati equations:

$$
\begin{gathered}
\left(\widehat{A}_{i}-K_{i} \widehat{C}_{i}\right) D_{i}+D_{i}\left(\widehat{A}_{i}-K_{i} \widehat{C}_{i}\right)^{T}+\sum_{j=1}^{N} \pi_{i j} D_{j}+I_{n}=0 \\
\left(\widehat{A}_{i}-K_{i} \widehat{C}_{i}\right) G_{i}+G_{i}\left(\widehat{A}_{i}-K_{i} \widehat{C}_{i}\right)^{T}+\sum_{j=1}^{N} \pi_{i j} G_{j}+K_{i} K_{i}^{T}=0 .
\end{gathered}
$$

According to the above analysis, seeking a maximum upper bound of noise uncertainty $(\Delta W, \Delta V)$ is equivalent to obtaining the optimal solution of $a, b$ :

$$
\begin{aligned}
\max & a \cdot b \\
\text { s.t. } & a \cdot \operatorname{tr}\left(D_{i}\right)+b \cdot \operatorname{tr}\left(G_{i}\right) \leq r+\max _{j \in S} \operatorname{tr}\left(Q_{j}\right)-\operatorname{tr}\left(Q_{i}\right), \quad a \geq 0, b \geq 0, i \in S .
\end{aligned}
$$

Thus, seeking the optimal solution $a, b$ is transformed to a nonlinear programming problem with linear inequalities' constraints. Now, we discuss how to find the analytical solution of such problem. 


\subsection{Analytical Solution}

Since $\Xi=\{(\Delta W, \Delta V)\}$ is a compact convex set and the inequalities' constraints in (3.10) compose a compact set on which $a \cdot b$ is defined as a continuous function, thus the nonlinear programming problem must have optimal solution $a^{*}, b^{*}$ and the existence of solution is proved. Next, we will seek the analytical solution $a^{*}, b^{*}$.

Decompose the original nonlinear programming problem (see (3.10)) into $N$ subproblems:

$$
\begin{aligned}
\max & a_{1} \cdot b_{1} \\
\text { s.t. } & a_{1} \cdot \operatorname{tr}\left(D_{1}\right)+b_{1} \cdot \operatorname{tr}\left(G_{1}\right) \leq r+\max _{j \in S} \operatorname{tr}\left(Q_{j}\right)-\operatorname{tr}\left(Q_{1}\right) \\
\max & a_{2} \cdot b_{2} \\
\text { s.t. } & a_{2} \cdot \operatorname{tr}\left(D_{2}\right)+b_{2} \cdot \operatorname{tr}\left(G_{2}\right) \leq r+\max _{j \in S} \operatorname{tr}\left(Q_{j}\right)-\operatorname{tr}\left(Q_{2}\right) \\
& \vdots \\
\max & a_{N} \cdot b_{N} \\
\text { s.t. } & a_{N} \cdot \operatorname{tr}\left(D_{N}\right)+b_{N} \cdot \operatorname{tr}\left(G_{N}\right) \leq r+\max _{j \in S} \operatorname{tr}\left(Q_{j}\right)-\operatorname{tr}\left(Q_{N}\right) .
\end{aligned}
$$

By using Lagrange method, we have the optimal analytical solution for each subproblem as

$$
\begin{aligned}
& a_{i}^{*}=\frac{r+\max _{j \in S} \operatorname{tr}\left(Q_{j}\right)-\operatorname{tr}\left(Q_{i}\right)}{2 \operatorname{tr}\left(D_{i}\right)}, \\
& b_{i}^{*}=\frac{r+\max _{j \in S} \operatorname{tr}\left(Q_{j}\right)-\operatorname{tr}\left(Q_{i}\right)}{2 \operatorname{tr}\left(G_{i}\right)} .
\end{aligned}
$$

Thus, the analytical solution for the original nonlinear programming problem (see (3.10)) is given as

$$
\begin{aligned}
& a^{*}=\min _{i \in S} a_{i}^{*}=\min _{i \in S}\left\{\frac{r+\max _{j \in S} \operatorname{tr}\left(Q_{j}\right)-\operatorname{tr}\left(Q_{i}\right)}{2 \operatorname{tr}\left(D_{i}\right)}\right\}, \\
& b^{*}=\min _{i \in S} b_{i}^{*}=\min _{i \in S}\left\{\frac{r+\max _{j \in S} \operatorname{tr}\left(Q_{j}\right)-\operatorname{tr}\left(Q_{i}\right)}{2 \operatorname{tr}\left(G_{i}\right)}\right\} .
\end{aligned}
$$

Remark 3.1. The analytical solution of the nonlinear programming problem is given by the above analysis; however, it is only an optimal solution for each subproblem. This analytical solution in (3.13) is local optimal, but global suboptimal. For the global optimal solution, we could only get the numerical solution using "fmincon" function in MATLAB software. The optimal analytical solution of such nonlinear programming problem is still an open problem in mathematics for further exploration.

Theorem 3.2. Consider Markovian jump system (2.6). If one adopts state estimator (2.7) and Kalman filter gain (2.8), there exists a maximum admissible compact set $\Xi$. As long as the uncertainty to noise covariance matrix satisfies $(\Delta W, \Delta V) \in \Xi$, the deviation of system state estimation error performance $\Delta J$ is within a given precision $r$. 
Remark 3.3. Take into account the existence of noise uncertainty, and the new noise covariance matrix is given as $(W+\Delta W, V+\Delta V)$. Thus, the former predesigned optimal Kalman filter gain $K_{i}$, which is deduced from stationary noise $(W, V)$, will no longer be optimal and may cause distortion of control signals. But, this does not mean that we need to redesign Kalman filter. According to the above analysis, if we can successfully limit noise uncertainty $(\Delta W, \Delta V)$ to be within an admissible compact set $\Xi$, the predesigned Kalman filter gain $K_{i}$ can still be effective though it is not optimal. Moreover, the deviation of estimation performance is ensured to be within a desirable precision $r$.

\section{Mini-Max Robust Filter}

Let $K_{1}^{*}, K_{2}^{*}, \ldots, K_{N}^{*}$ denote the standard Kalman filtering gain according to new noise covariance matrix pair $\left(W^{*}, V^{*}\right)=\left(W+\Delta W^{*}, V+\Delta V^{*}\right)$, which corresponds to the maximum admissible noise uncertainty $\left(\Delta W^{*}, \Delta V^{*}\right)$; thus $K_{1}^{*}, K_{2}^{*}, \ldots, K_{N}^{*}$ satisfy

$$
\begin{gathered}
A_{i} P_{i}^{*}+P_{i}^{*} A_{i}^{T}+\epsilon_{i} P_{i}^{*} E_{i}^{T} E_{i} P_{i}^{*}+\sum_{j=1}^{N} \pi_{i j} P_{j}^{*}+\frac{1}{\epsilon_{i}} H_{1 i} H_{1 i}^{T}+W^{*}=0, \\
K_{i}^{*}=\left(Q_{i}^{*} \widehat{C}_{i}^{T}+\frac{1}{\epsilon_{i}} H_{1 i} H_{2 i}^{T}\right)\left(\frac{1}{\epsilon_{i}} H_{2 i} H_{2 i}^{T}+V^{*}\right)^{-1}, \\
\left(\widehat{A}_{i}-K_{i}^{*} \widehat{C}_{i}\right) Q_{i}^{*}+Q_{i}^{*}\left(\widehat{A}_{i}-K_{i}^{*} \widehat{C}_{i}\right)^{T}+K_{i}^{*} V^{*} K_{i}^{* T}+\sum_{j=1}^{N} \pi_{i j} Q_{j}^{*}+\frac{1}{\epsilon_{i}} H_{1 i} H_{1 i}^{T}+W^{*}=0 .
\end{gathered}
$$

According to the least-square quality of standard Kalman filtering, we have

$$
\Delta J\left(K_{1}^{*}, K_{2}^{*}, \ldots, K_{N}^{*}, \Delta W^{*}, \Delta V^{*}\right) \leq \Delta J\left(K_{1}, K_{2}, \ldots, K_{N}, \Delta W^{*}, \Delta V^{*}\right) .
$$

On the other hand, with the establishment of Fact 1, there is

$$
\Delta J\left(K_{1}^{*}, K_{2}^{*}, \ldots, K_{N^{\prime}}^{*} \Delta W, \Delta V\right) \leq \Delta J\left(K_{1}^{*}, K_{2}^{*}, \ldots, K_{N^{\prime}}^{*} \Delta W^{*}, \Delta V^{*}\right)
$$

Thus, we have the following saddle-point inequality:

$$
\begin{aligned}
\Delta J\left(K_{1}^{*}, K_{2}^{*}, \ldots, K_{N}^{*}, \Delta W, \Delta V\right) & \leq \Delta J\left(K_{1}^{*}, K_{2}^{*}, \ldots, K_{N}^{*}, \Delta W^{*}, \Delta V^{*}\right) \\
& \leq \Delta J\left(K_{1}, K_{2}, \ldots, K_{N}, \Delta W^{*}, \Delta V^{*}\right) .
\end{aligned}
$$

By game theory, we have

$$
\min _{K_{i}} \max _{(\Delta W, \Delta V) \in \Xi} \Delta J\left(K_{1}, K_{2}, \ldots, K_{N}, \Delta W, \Delta V\right)=\max _{(\Delta W, \Delta V) \in \Xi} \min _{K_{i}} \Delta J\left(K_{1}, K_{2}, \ldots, K_{N}, \Delta W, \Delta V\right) .
$$

This means that the optimal estimation under the worst situation is a mini-max filter. It cannot only minimize the estimation performance under the largest noise uncertainty $\left(a^{*} I_{n}, b^{*} I_{m}\right)$, but can also ensure the deviation to be within a given precision $r$. For this reason, this Kalman filter design is a robust mini-max filter. 
Remark 4.1. Traditional Kalman filtering design is performed on the basis that noise covariance matrix is stationary and exactly known, and it will fail when the noise covariance matrix is unknown or has uncertainty. In our method, the filter design could be divided into two steps. Firstly, we design standard Kalman filter according to the stationary noise covariance matrix $(W, V)$, then via some technical methods such as noise control we impose the noise uncertainty to be within the given bound $\left(\Delta W^{*}, \Delta V^{*}\right)$, which could be presented in the form of nonstructural $\left(a^{*} I_{n}, b^{*} I_{m}\right)$. In practical dynamic process, when the noise uncertainty reaches the maximum, the ideal deviation of performance is $\Delta J\left(K_{1}^{*}, K_{2}^{*}, \ldots, K_{N}^{*}, \Delta W^{*}, \Delta V^{*}\right)$, and this deviation is less than the worst case $\Delta J\left(K_{1}, K_{2}, \ldots, K_{N}, \Delta W^{*}, \Delta V^{*}\right) \leq r$, which ensures the estimation of system states and control signals to beprecise to some extent, and the synthetical system to be robust and stable. For this reason, the Kalman filter design has robustness to noise uncertainty, and according to (4.5), this filter is also a mini-max filter .

\section{Simulation}

Consider the following two-mode Markovian jump system.

Let the system mode $r(t)=1$ be given by

$$
\begin{gathered}
\dot{x}_{1}=-0.6 x_{1}+0.5 x_{2}+0.01 \sin \left(x_{1}+x_{2}\right)+\omega_{1}^{0}, \\
\dot{x}_{2}=0.7 x_{1}+0.02 \cos \left(x_{1}-x_{2}\right)+\omega_{2}^{0}, \\
y=x_{1}+0.5 x_{2}+v^{0} .
\end{gathered}
$$

Let the system mode $r(t)=2$ be given by

$$
\begin{gathered}
\dot{x}_{1}=-x_{1}+0.6 x_{2}+0.02 \sin x_{2}+\omega_{1}^{0}, \\
\dot{x}_{2}=0.8 x_{1}-1.1 x_{2}+0.02 \cos x_{1}+\omega_{2}^{0} \\
y=x_{1}+v^{0}
\end{gathered}
$$

where uncertain state and measurement noise are $\omega^{0}=\left[\begin{array}{ll}\omega_{1}^{0} & \omega_{2}^{0}\end{array}\right]^{T}$ and $v^{0}$; its stationary covariance matrix is known as $W=\left[\begin{array}{cc}1 & 0 \\ 0 & 1\end{array}\right], V=1$; system mode transition matrix is $\Pi=$ $\left[\begin{array}{cc}-0.6 & 0.6 \\ 0.9 & -0.9\end{array}\right]$; the admissible bound of performance deviation is $r=0.3$.

The detailed algorithm is as follows.

(1) By applying Assumption 2.1, we have $A_{1}=\left[\begin{array}{cc}-0.6 & 0.5 \\ 0.7 & 0\end{array}\right], C_{1}=\left[\begin{array}{ll}1 & 0.5\end{array}\right], A_{2}=$ $\left[\begin{array}{cc}-1 & 0.6 \\ 0.8 & -1.1\end{array}\right], C_{2}=\left[\begin{array}{ll}1 & 0\end{array}\right], H_{11}=\left[\begin{array}{ll}0.1 & 0.2\end{array}\right]^{T}, E_{1}=\left[\begin{array}{ll}0.1 & 0.1\end{array}\right], H_{12}=\left[\begin{array}{ll}0.1 & 0.1\end{array}\right]^{T}, E_{2}=$ $\left[\begin{array}{ll}0.2 & 0.2\end{array}\right]$.

Notice that $\Delta C_{i} \equiv 0$; thus $H_{21}=H_{22}=0$.

(2) Solve (2.8) and get $Q_{1}, Q_{2}$ and $K_{1}, K_{2}: Q_{1}=\left[\begin{array}{l}2.6058 \\ 1.4207 \\ 1.4207\end{array}\right], Q_{2}=$ $\left[\begin{array}{ll}2.6573 & 1.4181 \\ 1.4181 & 2.8473\end{array}\right], K_{1}=\left[\begin{array}{l}0.8748 \\ 1.0921\end{array}\right], K_{2}=\left[\begin{array}{l}0.6567 \\ 0.4866\end{array}\right]$.

(3) Substitute the result to (3.10), using Lagrange method; the upper bound of noise uncertainty is given as

$$
a^{*}=0.1014, \quad b^{*}=0.1701
$$


(4) Let the new noise covariance matrix correspond to the maximum uncertainty:

$$
\begin{gathered}
W^{*}=W+\Delta W=W+a^{*} \cdot I_{2} \\
V^{*}=V+\Delta V=V+b^{*} \cdot I_{1} .
\end{gathered}
$$

(5) Repeat step (2), and we have the correspondent $Q_{1}^{*}, Q_{2}^{*}, K_{1}^{*}, K_{2}^{*}$ for new noise covariance matrix $\left(W^{*}, V^{*}\right)$ :

$$
\begin{gathered}
Q_{1}^{*}=\left[\begin{array}{ll}
2.7641 & 1.4417 \\
1.4417 & 3.2688
\end{array}\right], \quad Q_{2}^{*}=\left[\begin{array}{ll}
2.6788 & 1.4458 \\
1.4458 & 2.9011
\end{array}\right], \\
K_{1}^{*}=\left[\begin{array}{l}
0.8977 \\
1.3021
\end{array}\right], \quad K_{2}^{*}=\left[\begin{array}{l}
0.7012 \\
0.5041
\end{array}\right] .
\end{gathered}
$$

(6) Applying the robust Kalman filtering, there is saddle-point inequality:

$$
\begin{aligned}
\Delta J\left(K_{1}^{*}, K_{2}^{*}, \Delta W, \Delta V\right) & \leq \Delta J\left(K_{1}^{*}, K_{2}^{*}, \Delta W^{*}, \Delta V^{*}\right) \\
& =\max \left\{\operatorname{tr}\left(Q_{1}^{*}\right), \operatorname{tr}\left(Q_{2}^{*}\right)\right\}-\max \left\{\operatorname{tr}\left(Q_{1}\right), \operatorname{tr}\left(Q_{2}\right)\right\} \\
& =0.2337<0.3 \\
& =\Delta J\left(K_{1}, K_{2}, \Delta W^{*}, \Delta V^{*}\right) .
\end{aligned}
$$

From the above simulation, it is seen that with the noise uncertainty being limited within the upper bound $a^{*}, b^{*}$ via noise control, the deviation of system estimation performance is less than the admissible precision $r$. Because the analytical solution $a^{*}, b^{*}$ is global suboptimal, the deviation of system estimation performance $(0.2337)$ is obviously less than admissible precision (0.3), which means that this solution is a conservative one and the global optimal solution of $a, b$ could be a little greater than $a^{*}, b^{*}$. Thus, this method allows flexibility to the designer to some extent.

\section{Conclusion}

In this paper, robust Kalman filter for continuous-time Markovian jump nonlinear systems with uncertain noise is considered. For the stability of dynamic system when statistical information of noise is unavailable, a new design method is given by obtaining the maximum admissible bound of uncertainty to noise covariance matrix. Based on this, the deviation of system estimation performance is thus guaranteed to be within a given precision. Furthermore, the worst performance yielded by noise uncertainty can be minimized by this method since it is a mini-max robust filter. The analytical solution of the bound to noise uncertainty is also discussed in this paper, which is a global, suboptimal, and conservative solution using Lagrange method. The simulation results show the validity of this design.

\section{Acknowledgment}

This work has been funded by BK21 research project: Switching Control of Systems with Structure Uncertainty and Noise. 


\section{References}

[1] B. D. O. Anderson and J. B. Moore, Optimal Filtering, Prentice-Hall, Englehood Cliffs, NJ, USA, 1979.

[2] V. Poor and D. P. Looze, "Minimax state estimation for linear stochastic systems with noise uncertainty," IEEE Transactions on Automatic Control, vol. 26, no. 4, pp. 902-906, 1981.

[3] S. Sangsuk-Iam and T. E. Bullock, "Analysis of continuous-time Kalman filtering under incorrect noise covariances," Automatica, vol. 24, no. 5, pp. 659-669, 1988.

[4] Y.-L. Chen and B.-S. Chen, "Minimax robust deconvolution filters under stochastic parametric and noise uncertainties," IEEE Transactions on Signal Processing, vol. 42, no. 1, pp. 32-45, 1994.

[5] M. Mariton, Jump Linear Systems in Automatic Control, Marcel-Dekker, New York, NY, USA, 1990.

[6] Y. Ji and H. J. Chizeck, "Controllability, stabilizability, and continuous-time Markovian jump linear quadratic control," IEEE Transactions on Automatic Control, vol. 35, no. 7, pp. 777-788, 1990.

[7] X. Feng, K. A. Loparo, Y. Ji, and H. J. Chizeck, "Stochastic stability properties of jump linear systems," IEEE Transactions on Automatic Control, vol. 37, no. 1, pp. 38-53, 1992.

[8] F. Dufour and P. Bertrand, "The filtering problem for continuous-time linear systems with Markovian switching coefficients," Systems \& Control Letters, vol. 23, no. 6, pp. 453-461, 1994.

[9] C. E. de Souza, A. Trofino, and K. A. Barbosa, "Mode-independent $\mathrm{H}_{\infty}$ filters for Markovian jump linear systems," IEEE Transactions on Automatic Control, vol. 51, no. 11, pp. 1837-1841, 2006.

[10] S. K. Nguang and P. Shi, "Robust $\mathrm{H}_{\infty}$ output feedback control design for Takagi-Sugeno systems with Markovian jumps: a linear matrix inequality approach," Journal of Dynamic Systems, Measurement and Control, vol. 128, no. 3, pp. 617-625, 2006.

[11] J. Zhu, H. Xi, H. Ji, and B. Wang, "A robust adaptive controller for Markovian jump uncertain nonlinear systems with Wiener noises of unknown covariance," International Journal of Control, Automation and Systems, vol. 5, no. 2, pp. 128-137, 2007.

[12] P. Shi, E.-K. Boukas, and R. K. Agarwal, "Kalman filtering for continuous-time uncertain systems with Markovian jumping parameters," IEEE Transactions on Automatic Control, vol. 44, no. 8, pp. 1592-1597, 1999.

[13] M. S. Mahmoud, P. Shi, and A. Ismail, "Robust Kalman filtering for discrete-time Markovian jump systems with parameter uncertainty," Journal of Computational and Applied Mathematics, vol. 169, no. 1, pp. 53-69, 2004. 


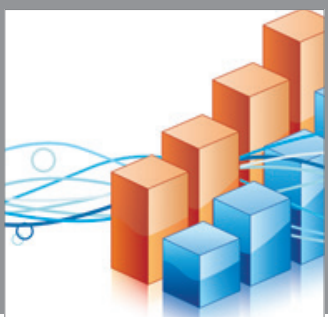

Advances in

Operations Research

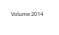

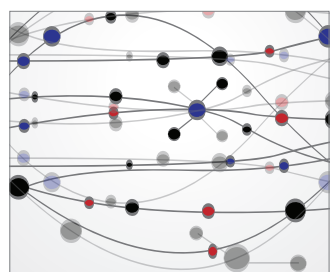

\section{The Scientific} World Journal
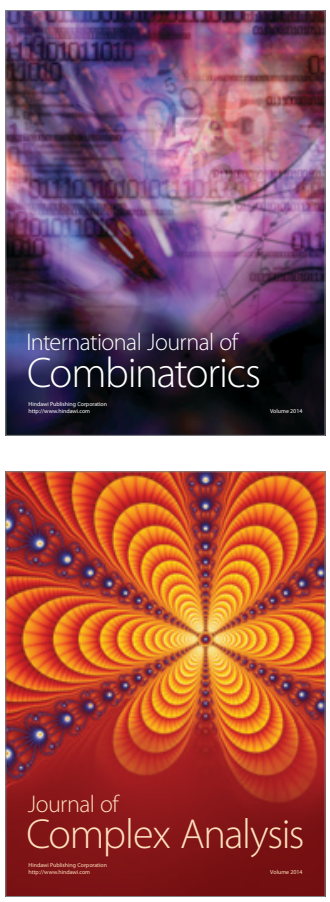

International Journal of

Mathematics and

Mathematical

Sciences
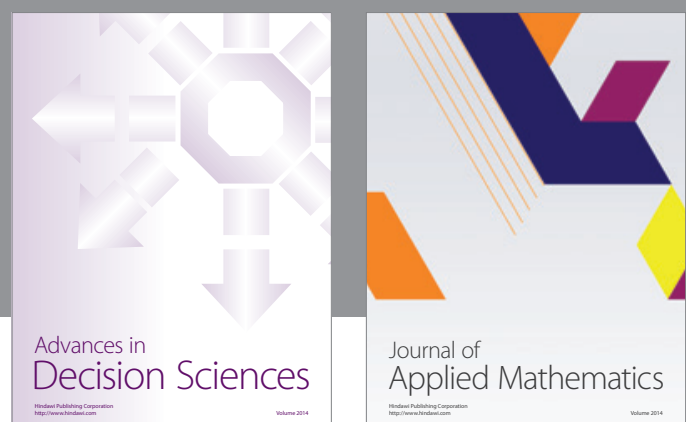

Journal of

Applied Mathematics
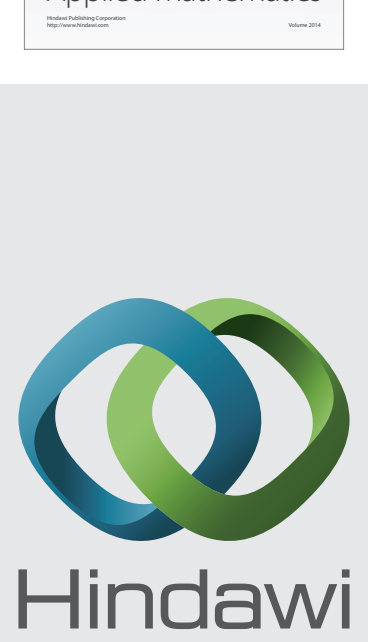

Submit your manuscripts at http://www.hindawi.com
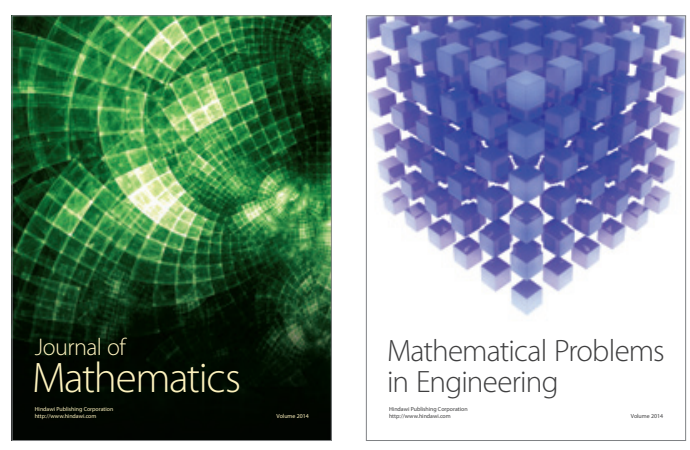

Mathematical Problems in Engineering
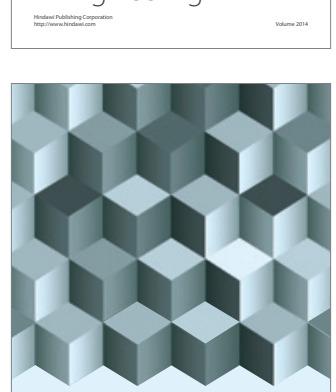

Journal of

Function Spaces
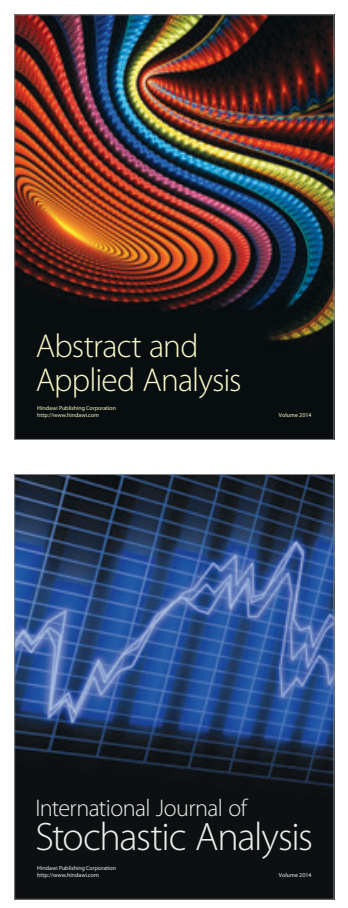

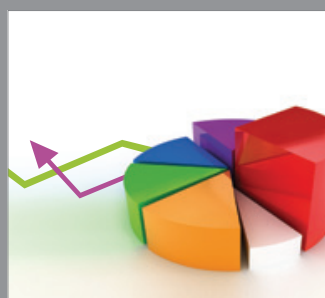

ournal of

Probability and Statistics

Promensencen
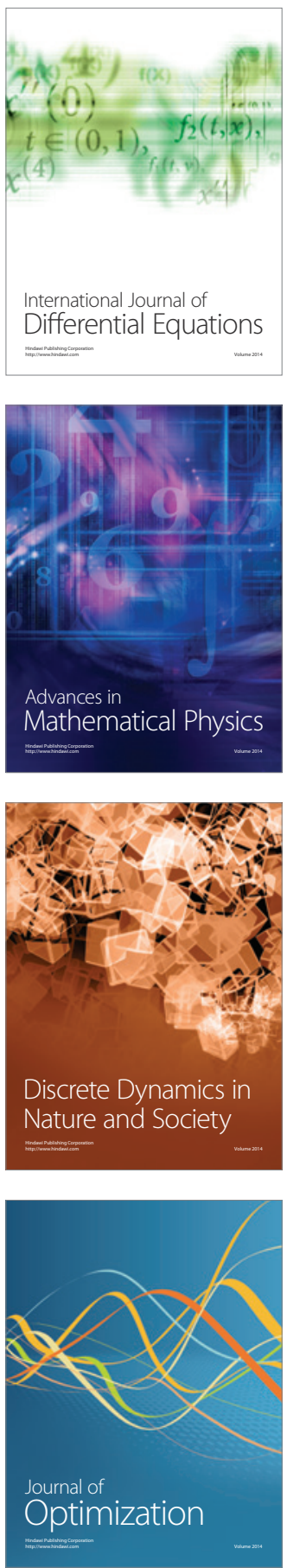